\title{
Solving DWF Dirac Equation Using Multi-splitting Preconditioned Conjugate Gradient with Tensor Cores on NVIDIA GPUs
}

\author{
Jiqun Tu \\ NVIDIA Corporation \\ Santa Clara, CA 95051, USA \\ jtu@nvidia.com \\ Chulwoo Jung \\ Brookhaven National Laboratory \\ Upton, NY 11973, USA \\ chulwoo@bnl.gov
}

\author{
M. A. Clark \\ NVIDIA Corporation \\ Santa Clara, CA 95051, USA \\ mclark@nvidia.com \\ Robert D. Mawhinney \\ Columbia University \\ New York, NY 10027, USA \\ rdm10@columbia.edu
}

\begin{abstract}
We show that using the multi-splitting algorithm as a preconditioner for the domain wall Dirac linear operator, arising in lattice QCD, effectively reduces the inter-node communication cost, at the expense of performing more on-node floating point and memory operations. Correctly including the boundary snake terms, the preconditioner is implemented in the QUDA framework, where it is found that utilizing kernel fusion and the tensor cores on NVIDIA GPUs is necessary to achieve a sufficiently performant preconditioner. A reduced-dimension (reduced- $L_{s}$ ) strategy is also proposed and tested for the preconditioner. We find the method achieves lower time to solution than regular CG at high node count despite the additional local computational requirements from the preconditioner. This method could be useful for supercomputers with more on-node flops and memory bandwidth than inter-node communication bandwidth.
\end{abstract}

\section{CCS CONCEPTS}

- Applied computing $\rightarrow$ Physics.

\section{KEYWORDS}

lattice QCD, domain wall fermion, GPU, tensor core, preconditioned conjugate gradient

\section{ACM Reference Format:}

Jiqun Tu, M. A. Clark, Chulwoo Jung, and Robert D. Mawhinney. 2021. Solving DWF Dirac Equation Using Multi-splitting Preconditioned Conjugate Gradient with Tensor Cores on NVIDIA GPUs . In Proceedings of ACM Conference (Conference'17). ACM, New York, NY, USA, 11 pages. https: //doi.org/10.1145/nnnnnnn.nnnnnnn

Permission to make digital or hard copies of all or part of this work for personal or classroom use is granted without fee provided that copies are not made or distributed for profit or commercial advantage and that copies bear this notice and the full citation on the first page. Copyrights for components of this work owned by others than ACM must be honored. Abstracting with credit is permitted. To copy otherwise, or republish, to post on servers or to redistribute to lists, requires prior specific permission and/or a fee. Request permissions from permissions@acm.org.

Conference'17, July 2017, Washington, DC, USA

(c) 2021 Association for Computing Machinery.

ACM ISBN 978-x-xxxx-xxxx-x/YY/MM...\$15.00

https://doi.org/10.1145/nnnnnnn.nnnnnnn

\section{INTRODUCTION}

\subsection{Situation and Motivation}

Quantum Chromodynamics (QCD) is the theory that describes the interaction between quarks and gluons, and accounts for most of the physical matter we encounter every day. Lattice QCD remains as the only viable first-principles method to calculate physical predictions from the non-linear theory. However, such calculations are extremely computationally demanding: consuming in excess of $10 \%$ of total cycles at large public supercomputer sites, such as NERSC [8].

The most computationally expensive part of full lattice QCD simulations is the solution of the Dirac equation. On a 4D spacetime grid, the discretized Dirac equation is a large sparse linear system and solving this system is required for both the Markov chain ensemble generation phase, where snapshots of the QCD vacuum are produced with the correct Boltzmann weight, and the measurement phase, where the expectation values (or ensemble averages) of desired QCD observables are calculated from these snapshots. Each snapshot, known as a gauge field configuration gives rise to a unique linear system with the Dirac equation.

Traditionally, Krylov sub-space solvers have been used to solve the Dirac equation and, as detailed below, the conjugate gradient (CG) algorithm [12] remains an optimal choice for some stencil discretizations of the Dirac operator. The convergence rate is limited by the condition number of the Dirac operator, which has become as large as $10^{8}$ in current simulations due to the small input quark masses required to reproduce physical phenomena, e.g., the correct physical pion mass ${ }^{1}$. Many approaches have been developed to accelerate the convergence rate and in this work we describe a method that is applicable for solving the Dirac equation for the domain wall fermions (DWF) discretization in the ensemble generation phase. While this discretization is very appealing from a physics point of view, due to its preservation of all of the continuum symmetries, it is this very property that makes the discretization more challenging to solve numerically.

An early step away from a traditional Krylov solver was a domain decomposition algorithm proposed for the so-called Wilson

${ }^{1}$ See table 2 for condition numbers of the operators used in this work. 
fermion discretization in [15]. This method uses a Schwarz Alternating Procedure (SAP) as a preconditioner for a generalized conjugate residual (GCR) solver. This was shown to be faster than the popular BiCGStab algorithm [10,26], but a useful implementation of this method has not been found for DWF. The additive Schwarz algorithm has been used for the Dirac equation inversion for various fermions [2, 21], but again not for DWF. Subsequently, marked improvements in the time to solution have been achieved via adaptive multigrid methods $[1,3,28]$, and deflation/eigenspace methods. Deflation/eigenspace methods generally calculate the eigenvectors for low-modes (those with small eigenvalues), and use these to reduce the condition number of subsequent solves. For DWF the EigCG algorithm [24] and the implicitly restarted Lanczos algorithm with Chebyshev polynomial accelerations method [22, 23] have been successfully deployed in current calculations. The significant setup costs with both adaptive multigrid and eigensolver methods render them more suitable for the measurement phase since this requires solving the same Dirac equation for many different right-hand sides (sources), allowing for amortization of these overheads. However, this is much more challenging for the generation phase where, at most, a half dozen linear solves are required per linear system.

For the ensemble generation phase, the Markov chain Monte Carlo method of choice is the Hybrid Monte Carlo (HMC) algorithm [9]. In this algorithm the Dirac equation is solved for each small change in the gauge field, and, because the gauge fields are changing, each solution is based on a different linear system. For Wilson fermions, combining the Schwarz preconditioner with HMC provided an efficient simulation algorithm [16]. While there have been various endeavors in applying multigrid methods for ensemble generation, for DWF these methods have come up short [17], owing to the difficulty in breaking even with respect to the setup costs. The fundamental issue is that since the gauge fields are evolving, the setup required for multigrid for one gauge field snapshot becomes less optimal for the next gauge field. Additional inherent difficulties arise with DWF, owing to the complex indefinite nature of the eigenspectrum of the linear operator [5]. ${ }^{2}$

For multi-node computers, lattice simulations divide the global space-time domain into non-overlapping sub-domains that are stored and computed locally on different nodes. While this increases the total compute capability, it requires communication between the processors. For CG, communication is required for each iteration of the solve: 1.) global collectives arising from vector inner products, and 2.) halo communication arising from satisfying data dependences in the stencil application. For Lattice QCD the latter is the rate limiter, and with which we concern ourselves with. The DWF operator when deployed on multi-node computers requires roughly 1 byte of local inter-node communication bandwidth for each flop (floating point operation) of on-node computing. ${ }^{3}$

While the architectures of recent generations of supercomputers have greatly increased the floating point operations per second (flops) on each node, as well as the local memory bandwidth, there has not been a corresponding increase in inter-node communication bandwidth. On some of the newest machines, for example the SUMMIT machine at ORNL, the communication has become

\footnotetext{
${ }^{2}$ This contrasts with the Wilson discretization which while non-Hermitian, generally has a positive real eigenspectrum, allowing for direct (non-normal) methods.

${ }^{3}$ The precise requirement varies with the size of the problem on each local node.
}

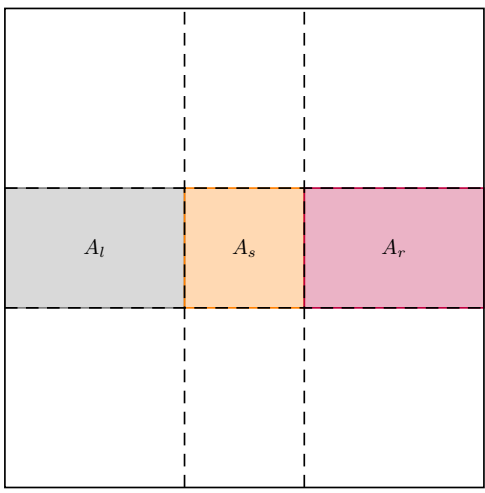

$A\left(=D^{\dagger} D\right)$

Figure 1: Decomposition of the matrix $A$, the solution vector $x$ and the right-hand-side vector $b$ into local parts on a node.

the bottleneck: when strong scaling the local computation time is between 10-20× less than the communication time and the large local computational resources are not utilized ${ }^{4}$.

In this work, we report on our investigation into an efficient solver for generating ensembles with DWF which consumes more local computation but less communication than standard CG. The cost of the local computation is reduced by an efficient implementation of the solver on NVIDIA GPUs utilizing the tensor cores, and an overall speedup in terms of time to solution is achieved.

\section{METHOD}

\subsection{Multi-splitting Algorithm}

In [20] the multi-splitting algorithm is proposed for solving generic linear systems. Compared to SAP, it does not require checkerboarding, using the current solution outside of each node as the Dirichlet boundary to perform local solutions. This acts as one iteration, and the solution is updated after each iteration, and the new boundary is communicated between nodes to ready the next iteration.

Following [14], assuming the equation to be solved is $A x=b$, at node $s$ the matrix $A$ and vectors $x$ and $b$ are decomposed according to figure 1 , where $x_{s}$ and $b_{s}$ are the parts that are locally stored. The original equation then turns into

$$
A_{s} x_{s}+A_{l} x_{l}+A_{r} x_{r}=b_{s} .
$$

The $A_{l} x_{l}+A_{r} x_{r}$ involves off-node vector parts and is calculated before each iteration with inter-node communication. Then for each iteration on each node the algorithm solves the equation

$$
A_{s} x_{s}=b_{s}-A_{l} x_{l}-A_{r} x_{r}
$$

locally for $x_{s}$. The updated solution $x_{s}$ will then be communicated to other nodes that need it. The local solves could be done concurrently on all nodes once the communication work to calculate $A_{l} x_{l}+A_{r} x_{r}$ is done.

\footnotetext{
${ }^{4}$ Aggregate single precision flops and total memory bandwidth of the 6 NVIDIA V100 GPUs on a SUMMIT node are 63 TFLOPS and $5.4 \mathrm{~TB} / \mathrm{s}$, respectively, while the bidirectional inter-node communication bandwidth is only $50 \mathrm{~GB} / \mathrm{s}$.
} 
The domain decomposition SAP algorithm and this algorithm treat the Dirichlet boundary differently and therefore the orders of local inversions are different: the former is multiplicative and requires checker-boarding, while the later is additive and does not require checker-boarding.

\subsection{Domain Wall Fermions}

The domain wall fermion (DWF) [13] formulation, or its variant Möbius DWF (MDWF) [6] which is used in this work, is one of several widely used discretizations of the continuum 4D Dirac operator. It suppresses the breaking of an important physical symmetry of QCD (chiral symmetry) at the cost of adding a fictitious fifth dimension of size $L_{s}$. The MDWF Dirac operator acts on pseudofermion vectors, which, on each $5 \mathrm{D}$ site, contain four spin and three color components of complex numbers to make a total of 24 real degrees of freedom per 5D site. The spin components represent quarks anti-commuting nature as fermions. The gauge fields are composed of $4 \mathrm{D}$ fields with unique 3-by-3 SU(3) matrices between each $4 \mathrm{D}$ lattice site. These matrices act in only in color space, i.e., they have no spin or fifth-dimension dependence.

Modern numerical implementations of MDWF utilize the fact that only the matrix elements that connect the even sites to odd sites and those connecting odd sites to even sites depend on the gauge field while the matrix elements that connect even sites to even sites and those connect odd sites to odd sites do not and are constant. Here the even-odd parity is defined by the $4 \mathrm{D}$ components of a site:

$$
\text { parity } \equiv(x+y+z+t) \quad \bmod 2 \text {. }
$$

In this even-odd order form the MDWF Dirac equation can be written as,

$$
\left(\begin{array}{cc}
M_{5} & M_{e o}^{4} \\
M_{o e}^{4} & M_{5}
\end{array}\right)\left(\begin{array}{l}
\psi_{e} \\
\psi_{o}
\end{array}\right)=\left(\begin{array}{l}
\phi_{e} \\
\phi_{o}
\end{array}\right)
$$

where $\psi$ is the desired solution vector, $\phi$ is the source vector, the subscript $e / o$ refer to even and odd sites and we have suppressed tensor indices for brevity. This is equivalent to solving the following even-odd preconditioned system (known as red-black Schur complement preconditioning in other parlance)

$$
\begin{aligned}
D \psi_{e}=\hat{\phi}_{e}, D & \equiv 1-M_{5}^{-1} M_{e o}^{4} M_{5}^{-1} M_{o e}^{4}, \\
\hat{\phi}_{e} & \equiv M_{5}^{-1} \phi_{e}-M_{5}^{-1} M_{e o}^{4} M_{5}^{-1} \phi_{o},
\end{aligned}
$$

Here 1 is the identity matrix, and $M_{e o / o e}^{4}$ includes the Wilson hopping (4D stencil) term $D_{x, y}^{w}$ that connects $4 \mathrm{D}$ space-time sites to their nearest neighbors,

$$
M_{o e / e o}^{4}=D_{x, y}^{w} M_{\phi}
$$

and $M_{5}$ and $M_{\phi}$ are constant matrices that are diagonal in the $4 \mathrm{D}$ Euclidean space-time dimensions. The Wilson-hopping term is given by

$$
D_{x, y}^{w} \equiv \sum_{\mu=1}^{4}\left[\left(1+\gamma_{\mu}\right) U_{x-\hat{\mu}, \mu}^{\dagger} \delta_{x-\hat{\mu}, y}+\left(1-\gamma_{\mu}\right) U_{x, \mu} \delta_{x+\hat{\mu}, y}\right],
$$

and it is here that the underlying gauge-field $U$ dependence comes in: the $\mathrm{SU}(3)$ matrices represent the coefficients of the nearestneighbor $D^{w}$ stencil. Since the radius $r=1$ of Wilson stencil, each application leads to a propagation, or hopping, of a non-zero contribution in each dimension and direction of size one. Exhaustive

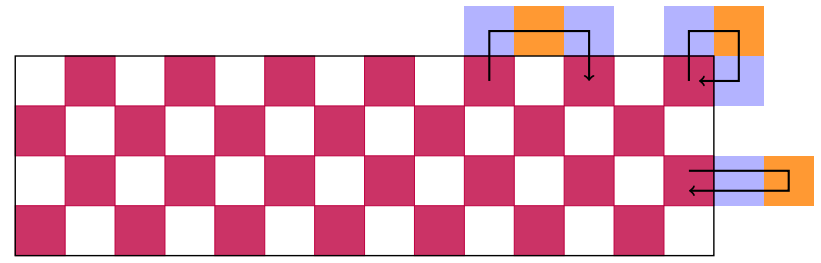

Figure 2: The normal operator $D^{\dagger} D$ has as many as four Wilson hopping terms (stencils). Enforcing Dirichlet boundary condition on it requires the inclusion of the snake terms, which are represented, as a $2 \mathrm{D}$ illustration, by the black arrows.

details of these matrices can be found in [6]. We note that given the $4 \mathrm{D}$ multi-processor decomposition only the $D^{w}$ contribution involves communication between processor sub-domains.

The CG algorithm requires the underlying matrix to be Hermitian and positive definite, while the matrix $D$ is complex indefinite. Hence Conjugate Gradient Normal Residual (CGNR) is often used: both sides of (5) are left multiplied by the Hermitian conjugate operator $D^{\dagger}$ and the resulting equation with the normal operator $D^{\dagger} D$ and the new RHS $D^{\dagger} \hat{\phi}_{e}$ is solved instead,

$$
D^{\dagger} D \psi_{e}=D^{\dagger} \hat{\phi}_{e} \text {. }
$$

\subsection{Dirichlet Boundary Condition on the 4-Hop Normal Operator}

There are four Wilson hopping terms, one in each $M_{e o / o e}^{4}$, in the normal operator $D^{\dagger} D$,

$$
D^{\dagger} D=\left[1-M_{5}^{-1} M_{e o}^{4} M_{5}^{-1} M_{o e}^{4}\right]^{\dagger}\left[1-M_{5}^{-1} M_{e o}^{4} M_{5}^{-1} M_{o e}^{4}\right] .
$$

To apply the multi-splitting algorithm to (9) Dirichlet boundary conditions are to be enforced on the normal operator $D^{\dagger} D$, i.e., the local part (the $A_{s}$ in (1)) of this normal operator needs to be constructed. As the vector content is distributed across the processors according to its $4 \mathrm{D}$ space-time location, this local part for $D^{\dagger} D$ includes snake terms that hop out of the boundary and hop back in as the various components in (10) are evaluated. Figure 2 illustrates this and gives some examples of the snake terms. These terms are truncated if Dirichlet boundary conditions are enforced on each of the four $M_{e o / o e}^{4}$ hopping terms sequentially. Using $\lfloor\bullet\rfloor$ to indicate applying Dirichlet boundary conditions,

$$
\left\lfloor M_{e o}^{4}\right\rfloor\left\lfloor M_{o e}^{4}\right\rfloor\left\lfloor M_{e o}^{4}\right\rfloor\left\lfloor M_{o e}^{4}\right\rfloor \neq\left\lfloor M_{e o}^{4} M_{o e}^{4} M_{e o}^{4} M_{o e}^{4}\right\rfloor .
$$

Our simulation results show that the correct inclusion of these snake terms is crucial to the convergence.

\subsection{Multi-splitting Algorithm as a Preconditioner of CG}

In [15] to achieve faster convergence the domain decomposition algorithm is eventually used as a preconditioner of GCR. In this work, since we are solving an Hermitian system, the multi-splitting algorithm is used as a preconditioner of CG. 
Pseudo-code for a generic preconditioned $\mathrm{CG}^{5}$ is shown in algorithm 1 , where we are solving $A x=b$ and $M$ is the preconditioner. The preconditioning step is marked with violet background. The overall convergence rate of preconditioned CG is estimated by the condition number of $A M^{-1}$. If the condition number of $A M^{-1}$ is smaller then that of the original matrix $A$, faster convergence rate will likely be achieved.

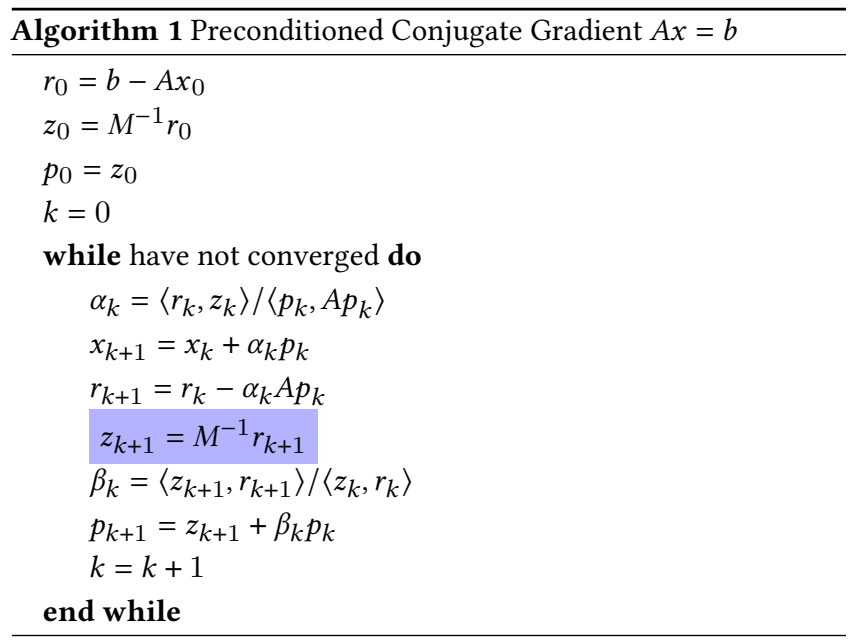

Now for this preconditioning step we use the multi-splitting algorithm to solve for $z_{k+1}$ in

$$
A z_{k+1}=r_{k+1} \text {. }
$$

To avoid inter-node communication, a zero initial guess $\left(x_{l}=x_{r}=\right.$ 0 ) is used in (2) and only the first iteration is performed. With $r_{k+1}$ as the RHS and $z_{k+1}$ the solution,

$$
A_{s} x_{s}=b_{s}-A_{l} x_{l}-A_{r} x_{r} \rightarrow A_{s} z_{k+1, s}=r_{k+1, s} .
$$

This is equivalent to using the local part of the matrix $A, A_{s}$, on each processor as the preconditioner $M$ in the preconditioned CG,

$$
M=\bigoplus_{s} A_{s}, s=\text { node index. }
$$

The local nature of $A_{s}$ makes it possible to perform the preconditioning step concurrently on all nodes without communication. We refer to this as multi-splitting preconditioned CG (MSPCG).

We note that while the multi-splitting algorithm can split the general matrix $A$ in a variety of ways, the splitting presented here, used as a preconditioner in CG, makes it equivalent to the additive Schwarz algorithm. We use the name MSPCG, as it is through the process of applying the multi-splitting algorithm to the MDWF Dirac equation that we realized the necessity of including the snake terms in the local matrix.

\subsection{The Effect of the Preconditioner on the Iteration Count}

The multi-splitting preconditioned CG is applied to solve Dirac equations on three lattice ensembles generated with Möbius domain

${ }^{5}$ For a reference of the preconditioned CG algorithm, see https://en.wikipedia.org/ wiki/Conjugate_gradient_method. wall fermions, using full lattice QCD with the quark masses set at their physical input values.

Regular CG is used to perform the linear solve in the preconditioning step. Instead of adopting a precision-based stopping condition, a fixed number of CG iterations, which will be referred as inner iterations, are performed for these preconditioning solves. The iterations performed in the overall preconditioned CG will be referred as outer iterations. In table 1 the number of outer iterations needed for the preconditioned CG to converge are reported on the different lattice ensembles, together with the stopping condition for the outer CG (precision) and the processor grid size used. The numbers of iterations to reach the same precision with standard CG are also included for comparison, where the inner iteration number is marked with plain.

We have two observations in terms of the effect of the preconditioner:

(1) Typically on these ensembles with 6 inner iterations the preconditioned CG reduces the outer iteration count by a factor of 3 . Beyond this, while more inner iterations can reduce the outer iteration count, there is only asymptotic improvement. This saturation is not surprising since increasing the inner iteration count is simply solving the preconditioning system with increasing accuracy, beyond which no further numerical benefit can be exploited.

(2) Executing a fixed number of inner CG iterations for the preconditioning inversion, instead of using a precision-based stopping condition, does not jeopardize the convergence of the outer CG despite the fact that CG, in principle, is not a flexible solver. This is true even when as few as 3 inner iterations are performed. This has previously been observed in [11].

Our results show that MSPCG reduces the number of outer iterations needed to solve the MDWF Dirac equation, reducing the inter-processor communication at the expense of performing local inner iterations for each outer iteration. The local inner iterations need to be sufficiently cheap compared to the outer matrix multiplication (the application of the matrix $A=D^{\dagger} D$ ) and corresponding BLAS (Basic Linear Algebra Subprograms) operations in order to achieve an overall speedup in terms of the time to solution.

As a consequence of this and the two observations, the inner iteration count is a parameter that can be tuned to achieve maximum speedup in the trade-off between inter-processor communication and local computation.

\section{IMPLEMENTATION OF THE METHOD ON NVIDIA GPUS IN QUDA}

The solver is implemented in QUDA, an open source library for performing calculations in lattice QCD on graphics processing units (GPUs), leveraging NVIDIA's CUDA platform [7]. With QUDA, we are able to fully utilize the global memory bandwidth, the different levels of cache, and, unique with this work, utilize the tensor cores available on recent NVIDIA GPUs, to achieve speedup. 
Table 1: Number of outer iterations need to converge the multi-splitting preconditioned CG for the lattice ensembles tested in this work. Inner iterations refers to the fixed number of CG iterations performed for the preconditioning inversion. Rows marked with plain indicate the iteration count for the same standard CG to converge. d.o.f. refers the number of real degrees of freedom of the linear system.

\begin{tabular}{|c|c|c|c|c|c|c|}
\hline lattice $4 \mathrm{D}$ volume & $L_{s}$ & d.o.f. & solver tolerance & processor grid size & inner iterations & outer iterations \\
\hline \multirow{4}{*}{$32^{3} \times 64$} & \multirow{4}{*}{12} & \multirow{4}{*}{$6.0 \times 10^{8}$} & \multirow{4}{*}{$10^{-8}$} & \multirow{4}{*}{$2^{3} \times 4$} & plain & 13594 \\
\hline & & & & & 3 & 9106 \\
\hline & & & & & 4 & 6020 \\
\hline & & & & & 6 & 5126 \\
\hline \multirow{4}{*}{$64^{3} \times 128$} & \multirow{4}{*}{10} & \multirow{4}{*}{$8.0 \times 10^{9}$} & \multirow{4}{*}{$10^{-10}$} & \multirow{4}{*}{$4^{3} \times 8$} & plain & 18092 \\
\hline & & & & & 6 & 6008 \\
\hline & & & & & 12 & 5083 \\
\hline & & & & & 18 & 4948 \\
\hline \multirow{2}{*}{$80^{2} \times 96 \times 192$} & \multirow{2}{*}{32} & \multirow{2}{*}{$1.1 \times 10^{11}$} & \multirow{2}{*}{$10^{-10}$} & \multirow{2}{*}{$4^{2} \times 8^{2}$} & plain & 16783 \\
\hline & & & & & 6 & 5719 \\
\hline
\end{tabular}

\subsection{An Efficient Implementation of the Preconditioner in QUDA with Tensor Core}

3.1.1 Efficient Stencil Application. As with other stencils acting on a regular grid, the most efficient implementation is generally obtained using a matrix-free formulation, encoding the action of the stencil on a grid, as opposed to an implementation as an explicit sparse-matrix-times-vector formulation. It is this approach that QUDA employs for all of the stencils it supports. With an arithmetic intensity of around 1-2 in single precision, it is a straightforward roofline analysis to see that an efficient implementation of the MDWF stencil will be memory-bandwidth bound on a single GPU.

Within a single node, with multiple GPUs, the bandwidth provided by the NVLink interconnect is ample to allow near perfect scaling over a node, however, when strong scaling on large-scale supercomputers, the performance of the matrix $A=D^{\dagger} D$ is typically limited by network bandwidth. This leaves little room for improvement from a software point of view without first innovating with respect to the choice of algorithm.

3.1.2 Capturing the Snake Terms. To apply the preconditioner, the snake terms must be included in order to enforce the correct Dirichlet boundary condition on (10). In our implementation the vector is padded in both directions of each partitioned dimension by size of two and the original field is placed at the center of the padded field while the pad region is initialized to zero. The operators in (10) of the full normal operator are then applied to the padded vector sequentially with zero Dirichlet boundary condition on the padded boundary. The four successive hops are propagated through the padded region. The padding size of two is sufficient, since with only four hops, in order to go back to the center region the snake terms are only allowed to hop out of the center region by as far as two steps. A 2D illustration is shown in figure 3.
This approach reuses the existing code for calculating the hopping term and the already highly optimized parallelizing strategies in QUDA. In order to avoid unnecessary work, we only update the minimum number of sites required to ensure correct evaluation; this means that a different number of sites are updated depending on which stage of the computation we are performing. For example, at the end of the computation, where we only desire the final result in the center region, there is no point updating the padded region.

By applying the hops successively the later hops reuse the results of the previous hops, which increases computation reuse compared to applying the individual 4-hop snake terms separately.

3.1.3 Kernel Fusion. A closer look at (10) gives the following series of operators

$$
\left[1-M_{\phi}^{\dagger} D^{w \dagger} M_{5}^{-\dagger} M_{\phi}^{\dagger} D^{w \dagger} M_{5}^{-\dagger}\right]\left[1-M_{5}^{-1} D^{w} M_{\phi} M_{5}^{-1} D^{w} M_{\phi}\right]
$$

Mathematically the operators are matrices to be left multiplied on the vector sequentially; numerically the operators are functions to be called to apply these multiplications; on the GPUs the operators are implemented as kernels to be launched. Conceptually each kernel carries out these three steps:

- Load the input vector from GPU memory into the registers;

- Perform computation with the data in the registers;

- Store the output vector from the registers to GPU memory.

Skipping the $[1-\cdots][1-\cdots]$ part in (15) for simplicity, we have 12 operators to be applied sequentially. Input of the kernels is exactly the output of the previous ones. Instead of storing the output all the way down to memory and then loading the very same data all the way up to the registers, a better strategy is to keep all the data in cache to avoid the memory traffic and latency from the repeated loading and storing from global memory. While this cache reuse would be automatically done by the GPUs in the L2 cache if the total memory footprint is less than the L2 cache 
Figure 3: A 2D illustration of using padded field to capture the snake terms. The orange and purple squares represent non-zero sites, and the white space represent zero sites. Black arrows represent the stencils relevant to the Dirichlet boundary condition. The stencil operators are not applied to the grey areas as they are either zero (as in 2)) or not needed for the output field at the center (as in 5) and 6)).
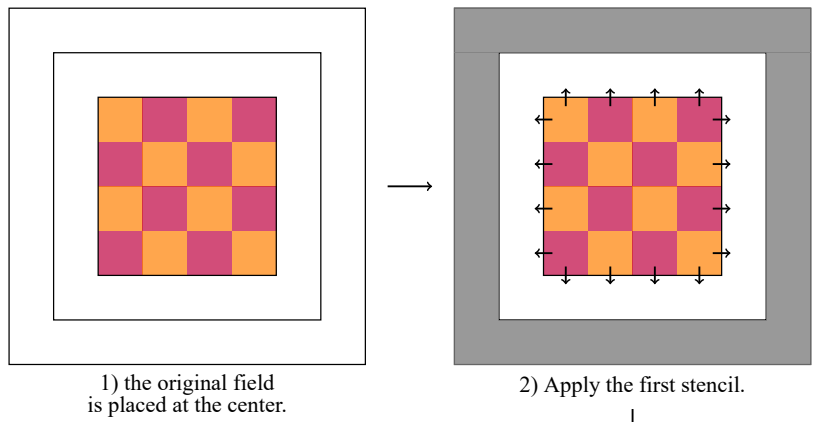

2) Apply the first stencil

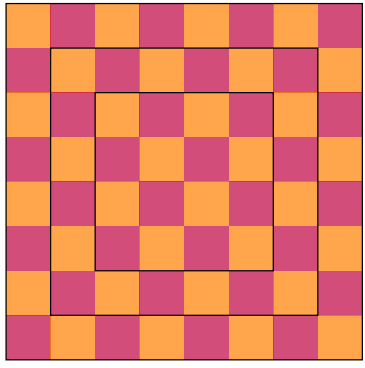

4) After applying the second stencil. $\downarrow$

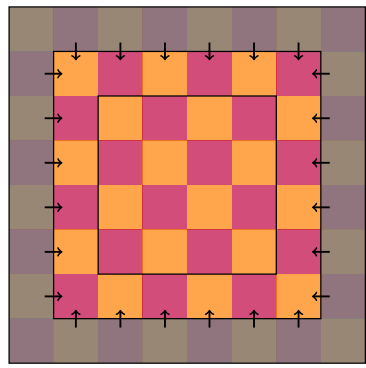

5) Apply the third stencil.

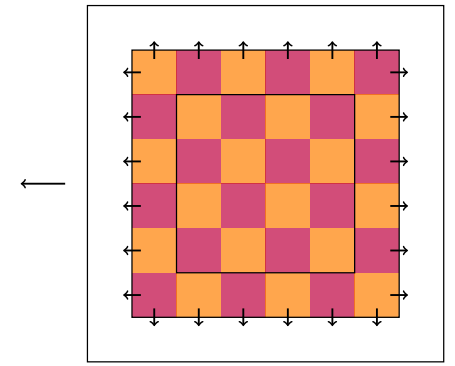

3) Apply the second stencil.

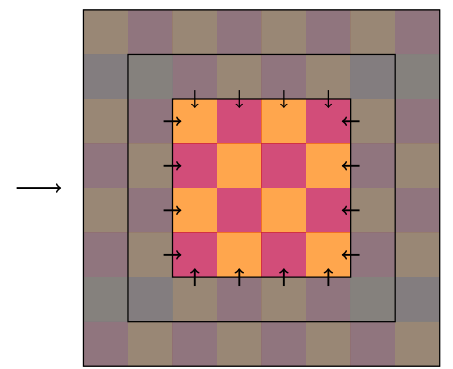

6) Apply the fourth stencil, and the output field is at the center.

size, the working set size of the problem at hand far exceeds the L2 capacity. In this work, data reuse is achieved by utilizing the structure of the matrices and the shared memory, utilizing explicit kernel fusion to increase the arithmetic intensity of the kernels.

The $D^{w}$ 's in (15) are matrices that hop in 4D space-time dimensions and are diagonal in the fifth dimension (4D stencil operators); the $M_{\phi}$ 's and $M_{5}^{-1}$ 's are matrices that only operate in the fifth dimension and are diagonal in the $4 \mathrm{D}$ space-time dimensions $(5 \mathrm{D}$ operators). As a result we can fuse an arbitrary number of 5D operators after a given application of $D^{w}$, so long as the extent of the fifth dimension is contained completely with in a single thread block, and hence can be communicated through shared memory.

Note that fusing multiple $D^{w}$ 's into one kernel is not feasible since it requires synchronization between thread blocks.

The kernels are deployed as a 2D grid of threads, with the $\mathbf{x}$ dimension corresponding to the $4 \mathrm{D}$ space-time points, and the $\mathbf{y}$ dimension corresponding to the 5D index. For each thread block the $4 \mathrm{D}$ operator $D^{w}$ is first applied and the results stored in shared memory; for the subsequent $5 \mathrm{D}$ operators, all of their input requirements will be what is held in the shared memory and so kernel fusion can be readily applied. The size of the thread block is chosen such that there is sufficient shared memory to hold the intermediate data, with autotuning of the block size further deployed to maximize the L2 hit rate for the initial 4D operator application.

Instead of launching 12 kernels for the 12 matrices in (15) only the following 5 fused kernels are launched:

$$
M_{\phi}^{\dagger} D^{w \dagger}, M_{5}^{-\dagger} M_{\phi}^{\dagger} D^{w \dagger}, M_{5}^{-\dagger} M_{5}^{-1} D^{w}, M_{\phi} M_{5}^{-1} D^{w}, M_{\phi} .
$$

3.1.4 Tensor Core. The $M_{5}^{-1}$ operator in (15) is a dense matrix multiplication in the fifth dimension, and the number of floating point operations needed scales as $O\left(L_{s}^{2}\right)$ for a parallel implementation. ${ }^{6}$ The computation required for the $M_{5}^{-1}$ application could become a bottleneck in the fused kernels, which are no longer trivially memory-bandwidth bound. This was indeed what we found in our early experiment, where we found that the preconditioner was rendered too expensive to improve the actual time to solution on the Piz Daint supercomputer (featuring the Pascal architecture).

As a remedy to this problem, the tensor cores and the HMMA (Half-precision Matrix Multiply and Accumulate) instructions, available on the NVIDIA Volta, Turing and Ampere GPUs, are used to speed up the application of this operator. Following the kernel fusion strategy described in the previous part the intermediate data is held in shared memory before applying $M_{5}^{-1}$. Since no global memory loads are required, this allows the HMMA instruction to be applied without being amortized by any global memory operation latency. As an illustration we show the how the HMMA instruction is applied in the $L_{s}=16$ case in figure 4 .

The half precision (IEEE FP16) has a much narrower representation range $\left(6.1 \times 10^{-5}\right.$ to 65504$)$ than that of single precision (IEEE FP32, $1.18 \times 10^{-38}$ to $3.4 \times 10^{+38}$ ). In order to avoid overflow and underflow, the maximum absolute value of the all the numbers in the thread block is found and the numbers are scaled such that they are representable in half precision before the HMMA instructions are applied. After the matrix multiplications the numbers are scaled back before being stored to memory as output. With a highly parallelized reduction and scaling code this overhead is negligible compared to the overall computation.

\subsection{Reduced Precision Strategies of Preconditioned CG}

QUDA supports 8-bit and 16-bit fixed-point formats as part of the effort to speed up memory-bandwidth limited kernels. For these kernels the floating point numbers are stored as 8-bit and 16-bit signed integers, together with a single-precision floating point number as the scale for every color-spinor $(\operatorname{color}(3) \times \operatorname{spinor}(4) \times$

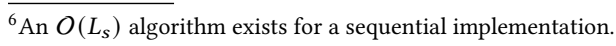


Figure 4: An illustration of how the $M_{5}^{-1}$, with $L_{s}=12$, and the number of 4D space-time sites dealt with in the CUDA thread block ("4d") being 16, is applied using the HMMA instruction. The matrix multiply is of shape " $48-$-by- 48 " $\times$ " $48-$ by- 96 " = " 48 by- 96 ", and is performed in the sub-block form of " 16 -by- 16 " $+=$ " 16 -by- 16 " $\times$ " 16 -by- 16 " with tensor cores as shown in the figure. Note that sizes of the shapes shown here are not to scale.

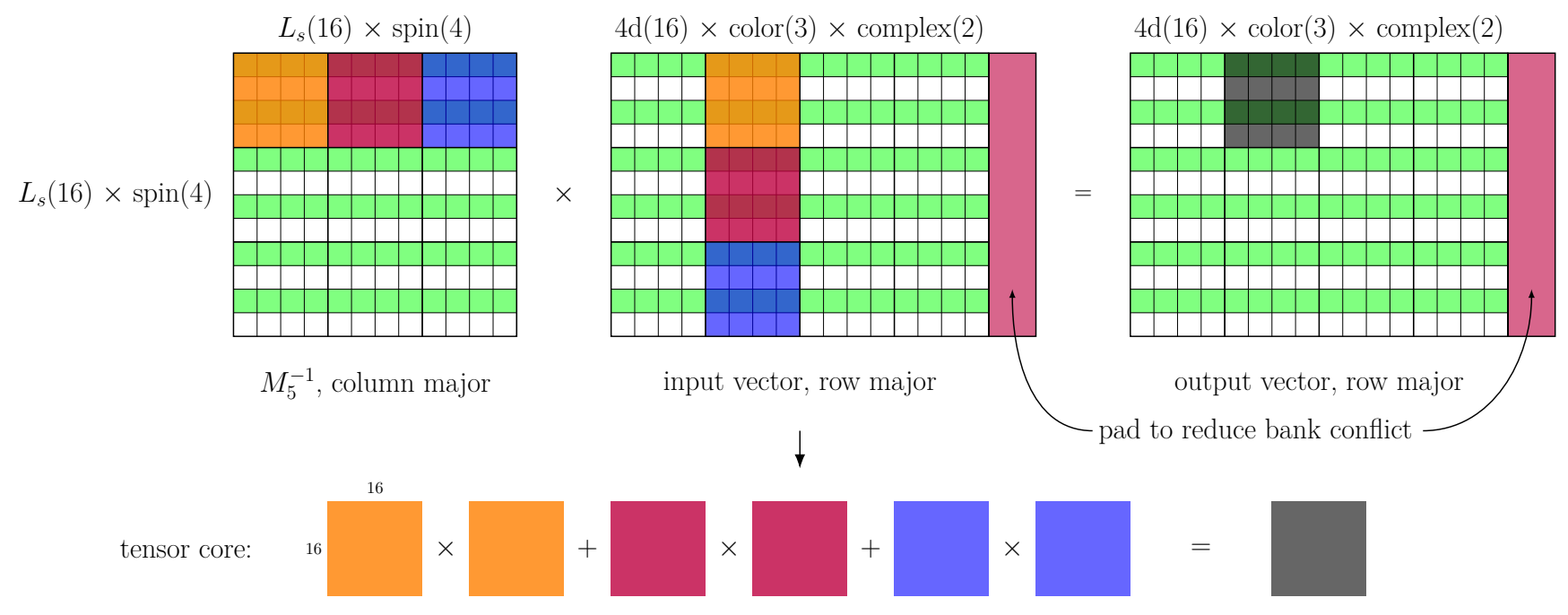

complex $(2)=24$ real numbers):

$$
\text { floating point }=\frac{\text { integer }}{\text { integer-limit }} \times \text { scale }
$$

In the kernels these fixed-point numbers are unpacked into single precision floating point numbers. Once they are loaded into registers the computations are done in single precision.

In our simulation most of the outer preconditioned CG iterations are performed with lower precision (16-bit fixed-point format) matrix multiplication and BLAS operations. For the solution to achieve higher precision (double precision) a reliable update strategy is deployed: the floating point error accumulated in lower precision during the lower-precision iterations is corrected with higher-precision matrix multiplication and BLAS operations when a certain condition (detailed in [27]) is reached.

For the inner preconditioner itself, the matrix multiplication and BLAS operations use the 8-bit and 16-bit fixed-point formats. For the fused tensor-core kernels the numbers are first loaded in fixedpoint formats, unpacked to single-precision floating point numbers for applying the stencils, then scaled and converted to half-precision floating point for applying the tensor-core instructions before being packed and stored in fixed-point format. Preconditioning kernels with 8-bit fixed-point format runs faster due to its lower memory traffic but there is a noticeable impact on the quality of the preconditioner, leading to increased outer iterations compared to the 16-bit case. The choice between 8-bit and 16-bit preconditioner depends on their overall effect on time to solution.

The inexact nature of lower precision also necessitates the use the of the Polak-Ribière ${ }^{7}$ version of the $\beta_{k}$ update in the preconditioned $\mathrm{CG}$, i.e.,

$$
\beta_{k}=\left\langle z_{k+1}, r_{k+1}-r_{k}\right\rangle /\left\langle z_{k}, r_{k}\right\rangle
$$

\footnotetext{
${ }^{7}$ See https://en.wikipedia.org/wiki/Conjugate_gradient_method.
}

\subsection{Accelerate the preconditioner with reduced- $L_{s}$ operators}

In the previous sections we have described our efforts on minimizing the cost of applying the local preconditioner on the technical side by efficiently utilizing the memory and cache bandwidth, as well as the tensor cores. In this section we detail our attempts to minimize the preconditioning cost on the algorithm side.

The kernels launched as part of the preconditioning are predominantly either global-memory-bandwidth bound, or L2-cachebandwidth bound for GPUs with large cache ${ }^{8}$. The time spent on the execution of these kernels is roughly proportional to the local $5 \mathrm{D}$ volume of the underlying vector. Thus, the cost of the preconditioning solve could be reduced if the fictitious fifth dimension $L_{S}$ is shrunk, and if this reduced- $L_{S}$ operator still captures the essential spectral qualities of the preconditioner, an overall speedup could be achieved. Such an approach would be a simplified two-grid algorithm applied in the fifth dimension.

The idea of using reduced- $L_{s}$ MDWF operators to accelerate the linear solvers has been explored in the Möbius accelerated domain wall fermion (MADWF) algorithm [29], where the domainoverlap equivalence [4] has been used to transform between domain wall systems with different $L_{s}$. In our case, however, this approach inflicts too high a numerical cost, since MADWF requires additional nested linear solves.

Our simplified approach proceeds as follows. Assuming the original Dirac operator with the original $L_{s}$ is $M$ (and the Dirac operator with reduced- $L_{s}$, denoted as $\hat{L}_{s}$, is $\hat{M}$ ) the original preconditioning step in preconditioned CG is

$$
z=M^{-1} r .
$$

\footnotetext{
${ }^{8}$ For example, the Ampere A100 GPU has a large L2 cache of $40 \mathrm{MiB}$
} 
Note that the inversion in $M^{-1}$ is performed with CG with a small fixed number of iterations. It is to be replaced with the reduced- $L_{s}$ version

$$
z=\left(T^{\dagger} \hat{M}^{-1} T+\mu \cdot 1\right) r,
$$

where the matrix $T$ transforms a vector with $L_{s}$ into a vector with $\hat{L}_{s}$, and the corresponding matrix $T^{\dagger}$ transforms a vector with $\hat{L}_{S}$ into a vector with $L_{s}$. This transform matrix $T$ acts on the vector as

$$
[T v]_{s, \mu}=\sum_{t, v}[T]_{s t, \mu v}[v]_{t, v}, t=1,2, \cdots, L_{s}, s=1,2, \cdots, \hat{L}_{s},
$$

where $\mu, v$ are spin indices. $T$ determines the transformation between different $L_{s}$ 's and a good $T$ reduces the cost of applying the preconditioning inversion while still managing to reduce the outer iteration count. The matrix $T^{\dagger} \hat{M}^{-1} T$ is Hermitian but not necessarily positive definite: zero modes exist since its rank is strictly smaller than the dimension of the vector $r$, if $\hat{L}_{s}<L_{s}$. The additional factor $\mu \cdot 1$ is inserted to suppress these zero modes, and its appearance is necessary to make the reduced- $L_{s}$ preconditioned CG converge.

In our approach $T$ is determined with a method that shares the idea of machine learning: the matrix $T$ is trained such that it minimizes the loss function of

$$
\chi^{2}=\sum_{i}\left|M \cdot\left(T^{\dagger} \hat{M}^{-1} T+\mu \cdot 1\right) \cdot v_{i}-v_{i}\right|^{2},
$$

where the $v_{i}$ 's are a set of vectors that have significant share of lowmode components of $M$. In our simulations the $v_{i}$ are the residual vectors $r_{k}$ in a preconditioned inversion with the original operators with the original $L_{s}$. In the case where $L_{s}=\hat{L}_{s}, M$ would be the same as $\hat{M}$ and the optimal $T$ would be the identity matrix, and the optimal $\chi^{2}$ would be zero. With the cost function $\chi^{2}$ defined and the its derivatives with respect to $T$ analytically available, $T$ is trained with the line search method $^{9}$ to minimize $\chi^{2}$. The minimization convergence is accelerated with the momentum method [25].

The value of the suppression factor $\mu$ is predefined for the minimization process and its choice is critical to the time-to-solution speed up of the algorithm. $\mu$ is tuned such that the resulting trained $T$ reduces the most outer CG iteration count ( $\mu$ being too large makes $T^{\dagger} \hat{M}^{-1} T+\mu \cdot 1$ close to an identity matrix) while is still able to suppress the zero modes of $T^{\dagger} \hat{M}^{-1} T$.

The transform matrix $T$ is only non-trivial in the spin components and the fifth dimension coordinates; and is trivial (diagonal) in the color degrees of freedom. Thus the $T$ trained on a single gauge field configuration can be used to accelerate linear solves performed on other configurations of the same ensemble, which amortizes the cost of training $T$.

\section{RESULTS}

The multi-splitting preconditioned CG, together with the reduced$L_{S}$ (machine learning) technique, is applied to the following two cases, both on SUMMIT:

- Solving MDWF Dirac equations for the light quark determinants in the evolution phase of the RBC/UKQCD collaborations' RBC96 lattice. The results are shown in figure 5 and

${ }^{9}$ For a reference of the line search method, see https://en.wikipedia.org/wiki/Line search. table 3. On 256 SUMMIT nodes MSPCG is able to achieve a $51 \%$ speedup over standard CG.

- Solving $\operatorname{MDWF}\left(L_{s}=12\right)$ Dirac equations for the light quark correlators in the measurement phase of the CalLat collaborations' CAL64 lattice $[18,19]$. The results are shown in figure 6 and table 4. On 64 SUMMIT nodes MSPCG is able to achieve a $31 \%$ speedup over standard CG.

Detailed information about the two lattices is shown in table 2.

The results show the reduced- $L_{s}$ operator, with the trained transform matrix $T$, is able to reduce the outer iteration count as a preconditioner for almost as much as the original operator is able to do. It has little to no effect on the overall convergence of the preconditioned CG.

Note that the transform matrix $T$ is trained with the set of vector $v_{i}$ generated from one of the configurations. The trained $T$ is then used on another configuration: this verifies that the training of $T$ is independent of which gauge configuration the inversions are performed on. Once $T$ is trained, it can be used on any other configurations of the same physical ensemble.

\section{CONCLUSION}

We have proven the feasibility of using local preconditioning to improve the multi-node scaling of lattice QCD simulations with (Möbius) domain wall fermions. MSPCG is able to reduce the (outer) iteration count by a factor of 3 with as few as 6 inner preconditioning iterations and achieve a $51 \%$ time-to-solution speedup on 256 SUMMIT nodes utilizing the tensor core on the V100 GPUs, together with various other optimizations and improvements. This is the first time the tensor cores, which are specifically designed to perform matrix-multiply operations, have been used to speed up lattice QCD simulations which are traditionally thought of as being bandwidth bound (whether memory or network).

Currently the performance of the preconditioning step is mainly limited by the GPU memory bandwidth and its cache efficiency, these are likely to be improve in the future at a faster rate than the the network bandwidth. Thus, we expect MSPCG to bring more significant speedup with this ever growing inequality between local and non-local memory bandwidth. The idea of using local preconditioning to improve overall scaling can also be applied to lattice QCD simulation with other fermion formulations and other scientific computing fields where halo communication is the limiting factor for multi-node scaling.

The acceleration that the tensor cores provide, applied through MSPCG, demonstrates that new algorithms can become feasible that would have otherwise led to a net slowdown. We expect this trend to continue going forward, where increased local tensor compute capability at low precision will give rise to increased algorithmic innovation to reduce the actual time to high-precision science.

\section{ACKNOWLEDGMENTS}

The authors wish to thank Duo Guo, Christopher Kelly and Norman Christ for the suggestions and comments, and want to thank Runzhi Wang for suggestion on using the line search method.

The full numerical implementation of algorithm is available in QUDA. Numerical experiments that contribute to this work are also done in CPS, Grid and Qlattice. 
Figure 5: $L_{2}$ residual as a function of (outer) iteration number for MSPCG (with and without reduced- $L_{S}$ acceleration) used on Dirac equation $\left(D^{\dagger} D x=y\right)$ linear solves on SUMMIT to a tolerance of $10^{-12}$ on the RBC96 lattice. $y$ is a Gaussian random source vector.
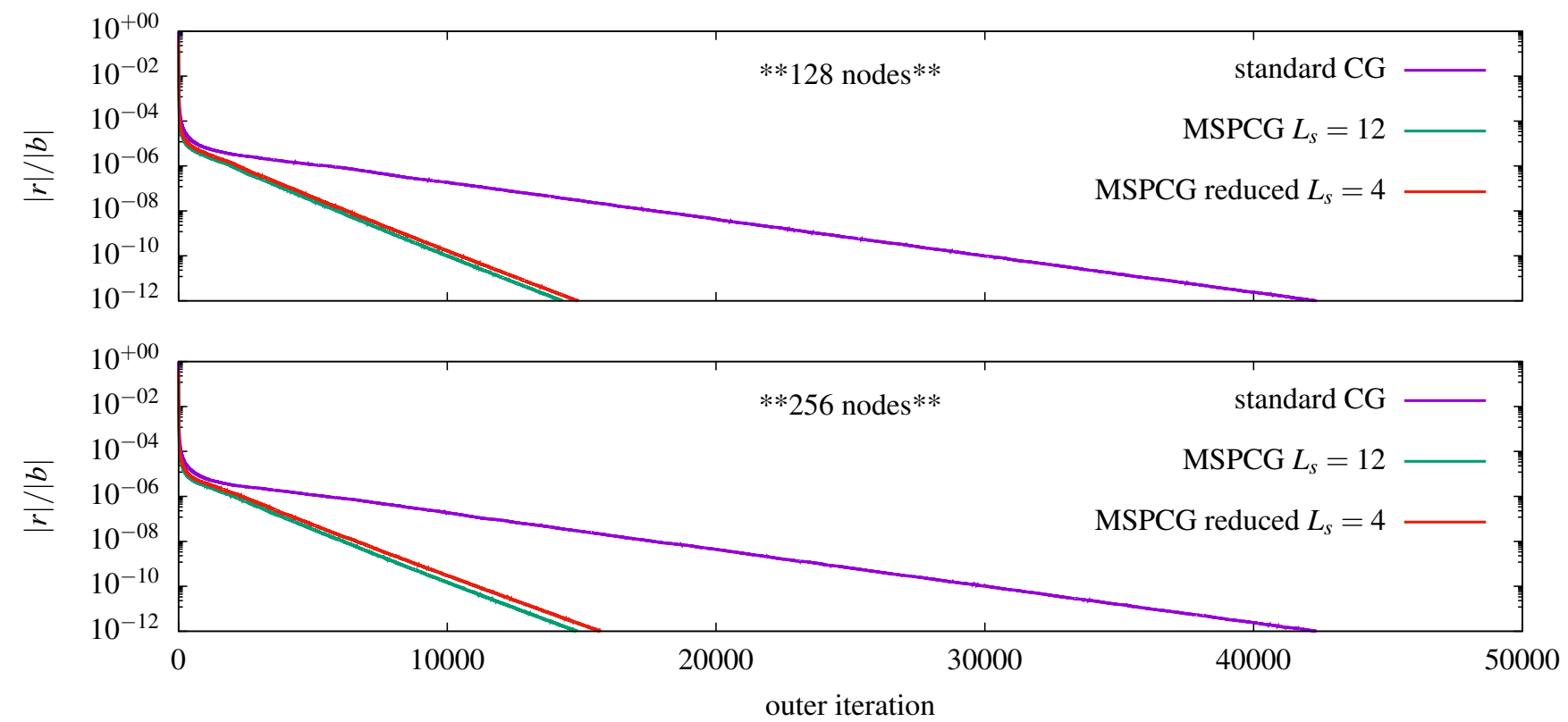

Figure 6: $L_{2}$ residual as a function of (outer) iteration number for MSPCG (with and without reduced- $L_{S}$ acceleration) used on Dirac equation $\left(D^{\dagger} D x=D y\right)$ linear solves on SUMMIT to a tolerance of $10^{-10}$ on the CAL64 lattice. $y$ is a random source vector.
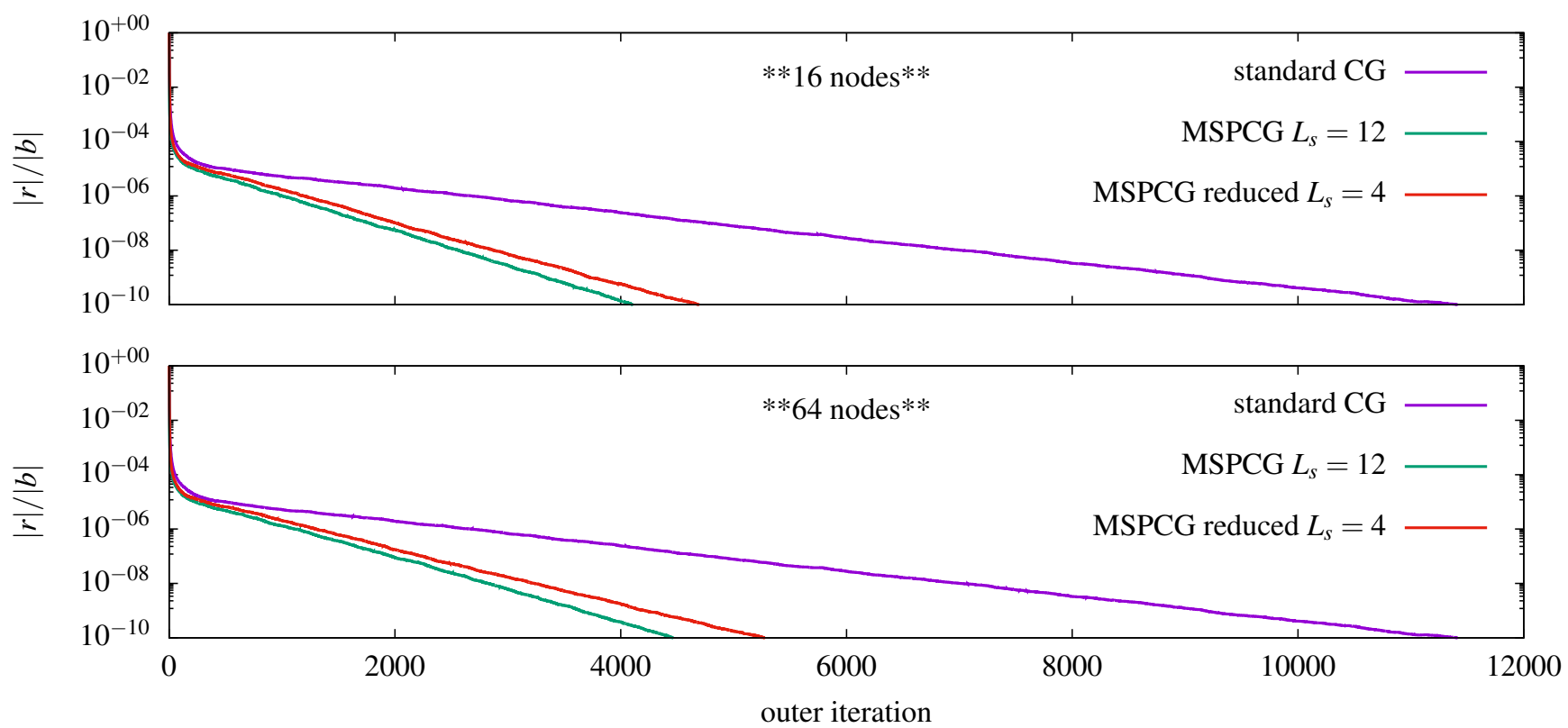
Table 2: Dirac operators used in the measurement and evolution phases, as well as other information of the two lattices used in this work. HISQ means Highly Improved Staggered Quark. $a^{-1}$ is the inverse lattice spacing of the lattices. d.o.f. refers to the number of real degrees of freedom of the linear system for solving MDWF Dirac equation, and $\kappa$ refers to the condition number of the MDWF Dirac operator with light input quark mass $m_{l}$.

\begin{tabular}{c|c|c|c|c|c|c|c}
\hline \hline label & 4 D volume & Dirac operator: measurement & Dirac operator: evolution & MDWF d.o.f. & $\kappa$ & $m_{l}$ & $a^{-1}[\mathrm{GeV}]$ \\
\hline RBC96 & $96^{3} \times 192$ & MDWF $L_{s}=12$ & $2+1$ flavor MDWF $L_{s}=12$ & $4.9 \times 10^{10}$ & $2.7 \times 10^{7}$ & 0.00054 & 2.8 \\
\hline CAL64 & $64^{3} \times 96$ & MDWF $L_{s}=12$ & $2+1+1$ flavor HISQ & $7.2 \times 10^{9}$ & $3.5 \times 10^{6}$ & 0.00152 & 2.2 \\
\hline \hline
\end{tabular}

Table 3: MSPCG (with and without reduced- $L_{s}$ acceleration) is used on Dirac equation $\left(D^{\dagger} D x=y\right)$ linear solves on SUMMIT to a tolerance of $10^{-12}$ on the RBC96 lattice. $y$ is a Gaussian random source vector. The times are given in units of seconds. The outer iterations are performed in 16-bit fixed-point format, while the residuals are corrected with reliable updates in double precision. The preconditioning is performed in 16-bit fixed-point format.

\begin{tabular}{c|c|c|c|c|c|c|c|c}
\hline \hline nodes & local volume & solver & inner iteration & $\mu$ & outer iteration & reliable update & time & speedup \\
\hline \multirow{2}{*}{128} & \multirow{3}{*}{$16 \times 24 \times 24 \times 24$} & CG & - & - & 42357 & 473 & 783.5 & 1 \\
& & MSPCG & 6 & - & 14264 & 166 & 744.5 & 1.05 \\
& & MSPCG+reduced- $L_{s}$ & 8 & 0.437 & 14849 & 173 & 542.2 & 1.44 \\
\hline \multirow{2}{*}{256} & \multirow{2}{*}{$16 \times 24 \times 12 \times 24$} & CG & - & - & 42342 & 473 & 577.6 & 1 \\
& & MSPCG & 6 & - & 14808 & 172 & 458.3 & 1.26 \\
\hline \hline
\end{tabular}

Table 4: MSPCG (with and without reduced- $L_{s}$ acceleration) is used on Dirac equation $\left(D^{\dagger} D x=D y\right)$ linear solves on SUMMIT to a tolerance of $10^{-10}$ on the CAL64 lattice. $y$ is a random source vector. The times are given in units of seconds. The outer iterations are performed in 16-bit fixed-point format, while the residuals are corrected with reliable updates in double precision. The preconditioning is performed in 16-bit fixed-point format.

\begin{tabular}{c|c|c|c|c|c|c|c|c}
\hline \hline nodes & local volume & solver & inner iteration & $\mu$ & outer iteration & reliable update & time & speedup \\
\hline \multirow{3}{*}{16} & \multirow{3}{*}{$32 \times 32 \times 16 \times 16$} & CG & - & - & 11383 & 133 & 217.1 & 1 \\
& & MSPCG & 6 & - & 4121 & 53 & 244.1 & 0.89 \\
& & MSPCG+reduced- $L_{s}$ & 6 & 0.850 & 4778 & 60 & 172.4 & 1.26 \\
\hline \multirow{2}{*}{64} & \multirow{2}{*}{$16 \times 16 \times 16 \times 16$} & CG & - & - & 11384 & 133 & 92.5 & 1 \\
& & MSPCG & 6 & - & 4464 & 57 & 90.2 & 0.98 \\
\hline \hline
\end{tabular}

The RBC96 lattice ensembles are generated by RBC/UKQCD collaborations, using the CPS code accelerated by QUDA on the SUMMIT supercomputer at Oak Ridge Leadership Computing Facility at the Oak Ridge National Laboratory, which is supported by the Office of Science of the U.S. Department of Energy.

R.D.M and J.T (while a student at Columbia University) are supported in part by U.S. Department of Energy contract No. DESC0011941. C.J. is supported in part by U.S. Department of Energy contract No. DE-SC0012704.

The CAL64 lattice ensembles are generated by the CalLat collaboration, using the MILC code accelerated by QUDA on the SUMMIT supercomputer at Oak Ridge Leadership Computing Facility at the Oak Ridge National Laboratory, which is supported by the Office of Science of the U.S. Department of Energy under Contract No. DE-AC05-00OR22725.

\section{REFERENCES}

[1] R. Babich, J. Brannick, R.C. Brower, M.A. Clark, T.A. Manteuffel, S.F. McCormick, J.C. Osborn, and C. Rebbi. 2010. Adaptive multigrid algorithm for the lattice Wilson-Dirac operator. Phys. Rev. Lett. 105 (2010), 201602. https://doi.org/10. 1103/PhysRevLett.105.201602 arXiv:1005.3043 [hep-lat]

[2] R Babich, M A Clark, B Joo, G Shi, R C Brower, and S Gottlieb. 2011. Scaling Lattice QCD beyond 100 GPUs. (2011). arXiv:1109.2935 
[3] J. Brannick, R.C. Brower, M.A. Clark, J.C. Osborn, and C. Rebbi. 2008. Adaptive Multigrid Algorithm for Lattice QCD. Phys. Rev. Lett. 100 (2008), 041601. https: //doi.org/10.1103/PhysRevLett.100.041601 arXiv:0707.4018 [hep-lat]

[4] R.C. Brower, H. Neff, and K. Orginos. 2006. Möbius Fermions. Nuclear Physics B - Proceedings Supplements 153, 1 (2006), 191 - 198. https://doi.org/10.1016/j. nuclphysbps.2006.01.047 Proceedings of the Workshop on Computational Hadron Physics.

[5] Richard C. Brower, M.A. Clark, Dean Howarth, and Evan S. Weinberg. 2020. Multigrid for Chiral Lattice Fermions: Domain Wall. (4 2020). arXiv:2004.07732 [heplat]

[6] Richard C. Brower, Hartmut Neff, and Kostas Orginos. 2005. Möbius fermions Improved domain wall chiral fermions. Nuclear Physics B - Proceedings Supplements 140, SPEC. ISS. (sep 2005), 686-688. https://doi.org/10.1016/j.nuclphysbps. 2004.11.180 arXiv:0409118 [hep-lat]

[7] M.A. Clark, R. Babich, K. Barros, R.C. Brower, and C. Rebbi. 2010. Solving Lattice QCD systems of equations using mixed precision solvers on GPUs. Comput. Phys. Commun. 181 (2010), 1517-1528. https://doi.org/10.1016/j.cpc.2010.05.002 arXiv:0911.3191 [hep-lat]

[8] Jack Deslippe. [n.d.]. Perlmutter - A 2020 Pre-Exascale GPU-accelerated System for NERSC. Architecture and Early Application Performance Optimization Results. Paper presented at the meeting of GPU Technology Conference 2019, San Jose CA. https://on-demand.gputechconf.com/supercomputing/2019/pdf/sc1919perlmutter---a-2020-pre-exascale-gpu-accelerated-system-for-nersc-architecture-and-early-application-performance-optimization-results.pdf

[9] S. Duane, A.D. Kennedy, B.J. Pendleton, and D. Roweth. 1987. Hybrid Monte Carlo. Phys. Lett. B 195 (1987), 216-222. https://doi.org/10.1016/0370-2693(87)91197-X

[10] A. Frommer, V. Hannemann, B. Nockel, T. Lippert, and K. Schilling. 1994. Accelerating Wilson fermion matrix inversions by means of the stabilized biconjugate gradient algorithm. Int. F. Mod. Phys. C 5 (1994), 1073-1088. https: //doi.org/10.1142/S012918319400115X arXiv:hep-lat/9404013

[11] Gene H. Golub and Qiang Ye. 1999. Inexact Preconditioned Conjugate Gradient Method with Inner-Outer Iteration. SIAM fournal on Scientific Computing 21, 4 (1999), 1305-1320. https://doi.org/10.1137/s1064827597323415

[12] M. R. Hestenes and E. Stiefel. 1952. Methods of conjugate gradients for solving linear systems. Fournal of research of the National Bureau of Standards 49 (1952) 409-436.

[13] Karl Jansen. 1996. Domain wall fermions and chiral gauge theories. Physics Report 273, 1 (1996), 1-54. https://doi.org/10.1016/0370-1573(95)00081-X arXiv:9410018 [hep-lat]

[14] Fabienne Jezequel, Raphaël Couturier, and Christophe Denis. 2012. Solving large sparse linear systems in a grid environment: The GREMLINS code versus the PETSc library. Fournal of Supercomputing 59, 3 (2012), 1517-1532. https: //doi.org/10.1007/s11227-011-0563-y

[15] Martin Lüscher. 2004. Solution of the Dirac equation in lattice OCD using a domain decomposition method. Computer Physics Communications 156, 3 (2004), 209-220. https://doi.org/10.1016/S0010-4655(03)00486-7 arXiv:0310048 [hep-lat]

[16] Martin Luscher. 2005. Schwarz-preconditioned HMC algorithm for two-flavour lattice QCD. Comput. Phys. Commun. 165 (2005), 199-220. https://doi.org/10. 1016/j.cpc.2004.10.004 arXiv:hep-lat/0409106

[17] Gregory Edward McGlynn. 2016. Advances in Lattice Quantum Chromodynamics Ph.D. Dissertation. Columbia U. https://doi.org/10.7916/D8T72HD7

[18] Nolan Miller et al. 2020. $F_{K} / F_{\pi}$ from $M \backslash$ " $\{$ o\}bius Domain-Wall fermions solved on gradient-flowed HISQ ensembles. Phys. Rev. D 102, 3 (2020), 034507. https //doi.org/10.1103/PhysRevD.102.034507 arXiv:2005.04795 [hep-lat]

[19] Nolan Miller et al. 2020. Scale setting the M\{\”o\}bius Domain Wall Fermion on gradient-flowed HISQ action using the omega baryon mass and the gradient-flow scale $w_{0} \cdot(112020)$. arXiv:2011.12166 [hep-lat]

[20] Dianne P O'Leary and R E White. 1985. Multi-Splittings of Matrices and Parallel Solution of Linear Systems. SIAM Journal on Algebraic Discrete Methods 6, 4 (1985), 630-640. https://doi.org/10.1137/0606062

[21] Yusuke Osaki and Ken-ichi Ishikawa. 2010. Domain Decomposition method on GPU cluster. (2010), 1-7. arXiv:1011.3318

[22] Y Saad. 1980. On the Rates of Convergence of the Lanczos and the Block-Lanczos Methods. SIAM 7. Numer. Anal. 17, 5 (1980), 687-706. https://doi.org/10.1137/ 0717059

[23] Eigo Shintani, Rudy Arthur, Thomas Blum, Taku Izubuchi, Chulwoo Jung, and Christoph Lehner. 2015. Covariant approximation averaging. Physical Review D - Particles, Fields, Gravitation and Cosmology (2015). https://doi.org/10.1103/ PhysRevD.91.114511 arXiv:1402.0244

[24] Andreas Stathopoulos and Konstantinos Orginos. 2010. Computing and Deflating Eigenvalues While Solving Multiple Right-Hand Side Linear Systems with an Application to Quantum Chromodynamics. SIAM fournal on Scientific Computing 32, 1 (2010), 439-462. https://doi.org/10.1137/080725532

[25] Ilya Sutskever, James Martens, George Dahl, and Geoffrey Hinton. 2013. On the importance of initialization and momentum in deep learning. In Proceedings of the 30th International Conference on Machine Learning (Proceedings of Machine Learning Research, Vol. 28), Sanjoy Dasgupta and David McAllester (Eds.). PMLR, Atlanta, Georgia, USA, 1139-1147. http://proceedings.mlr.press/v28/sutskever13. html

[26] H. A. van der Vorst. 1992. Bi-CGSTAB: A Fast and Smoothly Converging Variant of Bi-CG for the Solution of Nonsymmetric Linear Systems. SIAM 7. Sci. Stat. Comput. 13, 2 (March 1992), 631-644. https://doi.org/10.1137/0913035

[27] Henk A. van der Vorst and Qiang Ye. 2000. Residual Replacement Strategies for Krylov Subspace Iterative Methods for the Convergence of True Residuals. SIAM Journal on Scientific Computing 22, 3 (2000), 835-852. https://doi.org/10.1137/ s1064827599353865

[28] Azusa Yamaguchi and Peter Boyle. 2016. Hierarchically deflated conjugate residual. PoS LATTICE2016 (2016), 374. https://doi.org/10.22323/1.256.0374 arXiv:1611.06944 [hep-lat]

[29] Hantao Yin and Robert Mawhinney. 2012. Improving DWF Simulations: Force Gradient Integrator and the Mobius Accelerated DWF Solver. PoS Lattice 2011 (2012), 051. https://doi.org/10.22323/1.139.0051 